\title{
Principios y agenda en la política exterior china. Un análisis constructivista de los discursos de Xi
}

\author{
Principles and Agenda in Chinese Foreign \\ Policy. Constructivist Analysis over Xi's \\ Speeches
}

\section{Mariano Mosquera*}

\section{RESUMEN}

Los supuestos de la teoría constructivista en relaciones internacionales permiten modelar los principios y agenda del discurso de la diplomacia con características chinas. Este modelo pone énfasis en una orientación anti-hegemónica, en el respeto por la particularidad de los países y en una agenda múltiple. Sin embargo, en los discursos del Presidente chino Xi, y en comparación a la política doméstica y partidaria, solo el principio de "desarrollo pacífico" se destaca por su alta frecuencia y relaciones conceptuales. Además, la agenda en política exterior muestra una marcada concentración en economía y seguridad.

Palabras clave: Discurso - Diplomacia - Agenda - China - Xi Jinping.

\footnotetext{
Profesor e Investigador de la Universidad Sun Yat-sen en la República Popular China. Doctor en Ciencia Política de la Universidad Nacional de Córdoba en Argentina.Email: mosquera@ mail.sysu.edu.cn. Recibido: 27 de diciembre de 20I7. Aceptado: 13 de junio de 2018.
} 


\section{ABSTRACT}

Assumptions of the constructivist theory in international relations allow to model principles and agenda of the discourse of diplomacy with Chinese characteristics. This model emphasizes an anti-hegemonic orientation, respect for the particularity of the countries and a multiple agenda. However, in Chinese president Xi's speeches, and in comparison to domestic policy and partisan politics, only the principle of "peaceful development" stands out, because of its high frequency and its conceptual relations. Moreover, the agenda in foreign policy shows a clear concentration in economics and security.

Keywords: Discourse - Diplomacy - Agenda - China - Xi Jinping. 


\section{INTRODUCCIÓN}

El presente trabajo tiene como objetivo principal identificar la lógica de los principios de la política exterior de China en relación a su agenda temática y de actores. Para esto se han tomado como representativos los discursos seleccionados por el Presidente chino Xi (20I4) en su libro "La gobernación y administración de China" del año 20I4. Este se trata de un libro de 579 páginas, con 18 capítulos y 79 discursos y artículos fechados entre el is de noviembre del año 2012 y el I3 de junio de 20I4. Es decir, una recopilación oficial y actual que refleja las orientaciones del gobierno de la República Popular China.

Tres pilares se destacan en el libro: política doméstica, política exterior y política partidaria. Si bien los tres ejes serán referenciados de forma comparativa a lo largo del presente trabajo, en particular la política exterior china se refleja en 5 capítulos y 20 discursos. En el capítulo xi se define el marco conceptual de la cooperación china. Luego, el capítulo xII se centra en la relación de China con grandes potencias: Rusia, Estados Unidos y Europa. Seguidamente, el capítulo xIII abarca a los países vecinos de China, con discursos en Kazajistán e Indonesia, y conceptos articuladores como la "Ruta de la Seda". En el capítulo xIv hay tres discursos sobre regiones en desarrollo: África, América Latina y los países árabes. Finalmente, el capítulo xv se concentra en espacios institucionales de cooperación: BRICS (conformado por Brasil, Rusia, India, China y Sudáfrica), Foro воно ${ }^{1}$, G-20, Grupo de Shanghai ${ }^{2}$, APEC (en español, Foro de Cooperación Asia-Pacífico) y $\mathrm{CICA}^{3}$. En el anexo de este trabajo, se encuentra una identificación de dichos capítulos y discursos de política exterior del libro de Xi (20I4).

Dos abordajes complementarios se utilizan para alcanzar el objetivo de esta investigación: un abordaje teórico desde el constructivismo y uno metodológico desde el análisis de frecuencia léxica $(\mathrm{FL})$, semántica y concordancia.

En el primer caso, el constructivismo se trata de una corriente teórica, para el análisis de las relaciones internacionales, que ha puesto el acento en los significados y en los sentidos que son asignados sobre las acciones de los países en el escenario internacional (Vitelli, 20I4; Wendt, 1999; Hopf, 1998). De esta forma, analizaremos las prácticas discursivas chinas para identificar sus conceptos y sus relaciones. Esta es una forma de, entre otras posibles,

1 Foro económico constituido en el año 200I, es conocido como el "Davos de Asia".

2 Organización euroasiática dedicada a tratar temas de política, economía y seguridad. Creada en el año 2002, está compuesta por China, Kazajistán, Kirguistán, Rusia, Tayikistán y Uzbekistán.

3 Conference on Interaction and Confidence-Building Measures in Asia. Foro creado en el año I993, reúne a 26 países en temas de seguridad en Asia. 
interpretar la "visión del mundo" de China, sus acciones y las consecuencias sobre las acciones de otros actores.

En particular, identificaremos principios claves en su lógica de articulación conceptual y en relación a la agenda de temas y de actores. En este sentido, los principios se refieren a términos con orientaciones normativas (Beutel, 1965; Von Wright, 1963) o prescriptivas (Dworkin, 20I4). Mientras que la lógica de articulación conceptual se refiere a los distintos niveles de vinculación de los términos por relación semántica (Bach, 200I) y por concordancia (Sinclair, 199I), ya que estos principios suelen ser de tipo compuestos y, además, relacionados con temas y actores en un lenguaje descriptivo. De esta forma, se espera construir adecuadamente el sentido de los principios de la política exterior china junto con la identificación de su agenda (Aruguete, 2015).

Dado el abordaje teórico, que desarrollaremos en profundidad en el apartado segundo, la hipótesis de la presente investigación es que el discurso de política exterior de características chinas adopta una orientación constructivista.

Aquí se debe aclarar que la perspectiva de "características chinas" refiere a un enfoque con antecedentes de estudio y que la orientación constructivista se referirá a una particular construcción de los principios y la agenda en la política exterior china. Específicamente, principios que buscan evitar el control hegemónico en el escenario internacional, destacar las particularidades nacionales y el respeto por estas, y promover la cooperación como método. Mientras que la agenda adquiere patrones de multiplicidad. Por el contrario, no se espera, de forma comparada, encontrar tal construcción (como orientación) de principios y agenda en la política doméstica y partidaria. En este último caso, por la esperada preponderancia de normas e instituciones de control en una agenda de mayores características sociales y culturales.

Luego, en cuanto al abordaje metodológico, se realizará un estudio de frecuencia léxica (Henderson y Hewings, 1990) para la priorización de principios, temas y actores en el discurso chino. Se realizarán tanto procesos de separación de palabras vacías como procesos de identificación de términos ambiguos (Sinclair, 199I). Luego, se controlarán los principios y los temas con categorizaciones emergentes (Rodríguez Sabiote, Lorenzo Quiles y Herrera Torres, 2005). Seguidamente, el enfoque de términos claves (Biber, Conrad y Reppen, 1998) indica que solo serán considerados pertenecientes a los principios de política exterior aquellos términos que tengan FL no esperadas, en comparación a la política doméstica y a la política partidaria china.

A continuación, para un análisis relacional, los principios y la agenda serán trabajados con un análisis de concordancia, Key Word in Context o KwIC (Sinclair, 199I), análisis de distribución (Jacomy, Venturini, Heymann y Bastian, 2014) y autoridad (Kleinberg, 1997) y, finalmente, análisis comparados (Pe- 


\section{Mariano Mosquera}

Principios y agenda en la política exterior china. Un análisis constructivista de los discursos de $\mathrm{Xi}$

ters, 1998; Lijphart, 197I), para interpretar adecuadamente los resultados.

Así, en el primer apartado de esta investigación se revisan antecedentes académicos actuales que analizan la política exterior china, para contextualizar adecuadamente el debate sobre su discurso. Del mismo modo, se revelan antecedentes sobre enfoques constructivistas aplicados, que son de interés para el desarrollo de este traba- jo. En un segundo apartado se definen los conceptos centrales para la prueba empírica y el tercer apartado explicita los métodos utilizados para dicha prueba empírica. El cuarto apartado ofrece los resultados de la investigación. Finalmente, se presentan conclusiones y ejes de discusión que relacionan los hallazgos de la investigación con apreciaciones sobre la política exterior de China.

\section{Antecedentes}

El estudio sobre la política exterior de China es, en la actualidad, un área de creciente interés. Esto tiene como principal razón las diversas especulaciones sobre el crecimiento económico de China (Kotschwar, 20I4; Jenkins, 20I2) y su relación con orientaciones políticas de tipo hegemónicas (Morales Ruvalcaba, 20I7; Ikenberry, 2008). El debate es por demás complejo, debido a que a pesar del marcado incremento de poder nacional de China (Morales Ruvalcaba, 2015) este sigue siendo un país semiperiférico (Wallerstein, 2003a, 2003b; Arrighi y Drangel, 1986). Esto conlleva a diferentes tipos de análisis sobre el discurso chino en materia de política exterior.

El primero de ellos es que China tendría intenciones de afianzar un poder de tipo comunicativo-cultural (Serbin, 20I7) a través de su retórica (Corkin, 20I4) y en la forma de "soft power" (Nye Jr., 2004). Esto le permitiría a China superar dicha semiperiferia (junto con otras variables) e instalarse en el centro del sistema-mundo. En este sentido, "desde una perspectiva futura, para elevar su poder de discurso internacional, China necesita defender nuevas ideas y nuevos conceptos, acordes con la tendencia de los tiempos; participar activamente en influenciar la agenda internacional, y jugar un papel de liderazgo en el establecimiento de mecanismos internacionales, normas y orden. Aún más, China debe prestar más atención (...) en orden a formar conceptos comunes, consensos colectivos e identidades" (Yu, 20I7: I40). En términos generales, esta es una perspectiva que le impregna un sentido instrumental a los significados del discurso, ya que el fin último es afianzar el poder de tipo material. Esta es la visión predominante en lecturas neo-utilitaristas de las relaciones internacionales, como bien ha observado Ruggie (1998), donde "el ascenso del realismo y del neolibera- 
lismo institucionalista desde el año I980 y su convergencia alrededor de las premisas y preceptos neo-utilitaristas [propició que] los factores ideacionales, cuando han sido examinados, fueron modelados en términos estrictamente instrumentales, con utilidad o no para las propias unidades individuales, en su persecución de típicos intereses materiales" (Ruggie, 1998: 855).

Por otra parte, el segundo análisis destaca la particularidad discursiva china, que requiere de determinados esfuerzos de comunicación y entendimiento. Este enfoque supone el marco de la promoción de un sistema multipolar por parte de China (Pastrana y Vera, 20I7). En este último sentido se enmarca el propio libro de Xi (20I4). La nota del editor menciona en dicho libro: "A fin de responder a los intereses de la comunidad internacional y ayudarle a aumentar su entendimiento de los conceptos y el camino del desarrollo de China, así como sus políticas interior y exterior, la Oficina de Información del Consejo de Estado, la Oficina de Investigación de la Literatura del Partido Comunista de China y Ediciones en Lenguas Extranjeras, han compilado conjuntamente este libro: 'Xi Jinping: la Gobernación y Administración de China"” (Xi, 20I4: 3). Es decir, desde esta perspectiva, se trata de una diplomacia con características chinas. En este sentido, $\mathrm{Xi}$ ha dicho que China debe "conducir la diplomacia, destacando las características chinas y la visión de China" ${ }^{\text {. Asimis- }}$ mo, Liu (20I7) ha dicho que "la diplomacia con características chinas (...) sostiene las banderas de la paz, el desarrollo, la cooperación y el resultado ganar-ganar" (Liu, 20I7: 28). También, de forma ordenada, Su (20I7) plantea los ejes innovadores y característicos de esta diplomacia, con énfasis en su nueva fase bajo la presidencia de Xi: "El Partido abrió un nuevo capítulo en la construcción teórica de la diplomacia de características chinas: primero, el entendimiento de que el período de oportunidad estratégica de China se ha profundizado; segundo, que la estrategia fundamental de seguir el camino del desarrollo pacífico se ha clarificado; tercero, que la estrategia de beneficio mutuo y progreso ganar-ganar se ha propuesto; cuarto, que la visión de un mundo armonioso, siguiendo la paz y la prosperidad común, se ha defendido, y quinto, que los arreglos institucionales generales de la diplomacia de China han sido largamente explorados y mejorados" (Su, 20I7: 25). De esta manera, esta diplomacia de características chinas tendría como principal eje el evitar la hegemonía con la cooperación internacional, para el beneficio mutuo de los actores en el escenario internacional.

Así, el debate sobre el discurso de la política exterior de China puede trabajarse contraponiendo estas dos

4 "Conferencia central de trabajo relativa a los asuntos internacionales”, Beijing, 29 de noviembre de 2014 . 


\section{Mariano Mosquera}

Principios y agenda en la política exterior china. Un análisis constructivista de los discursos de $\mathrm{Xi}$

visiones desarrolladas anteriormente. En el primer caso, se trata de una perspectiva desde el neo-utilitarismo en relaciones internacionales; mientras que para el segundo, modelaremos una orientación constructivista en relaciones internacionales para la política exterior de características chinas. Algunas posiciones que podrían considerarse más intermedias en este debate, por ejemplo un análisis desde la teoría de la interdependencia en relaciones internacionales (Keohane, 2006; Keohane y Nye Jr., 1989), pueden también adecuarse como objeto de discusión en esta investigación, aunque exceden el presente desarrollo de este trabajo.

Finalmente, respecto del enfoque teórico y metodológico-empírico de esta investigación, mencionamos que Vitelli (20I4) ha destacado que nume- rosos estudios constructivistas han realizado pruebas empíricas, demostrando la relevancia de los significados en el accionar de los estados. Los estudios de Katzenstein (1996), Price y Tannenwald (1996), y Finnemore (1996), pueden ser mencionados como modelos de investigaciones empíricas en el marco del constructivismo. Así, el presente trabajo busca reconstruir con pruebas empíricas el discurso chino en materia de política exterior, esto como enfoque aplicado en el marco de la teoría constructivista. Este tipo de diseño de investigación, tanto teórico como metodológico, identifica principios de política exterior en relación a la agenda china en el escenario internacional, permitiendo así realizar aportes al ya mencionado debate central sobre la política exterior de China.

\section{Marco Teórico}

Si bien el constructivismo no se trata ya de una teoría nueva en el marco de los estudios en relaciones internacionales, sí se trata — como ha señalado Vitelli (20r4) - de una teoría de complejo abordaje. Esto último, sobre todo, si se piensa como marco aplicado a casos de política exterior. Es decir, una teoría de creciente relevancia, pero de difícil clasificación.

Sin entrar en los debates sobre las diversas perspectivas del constructivismo, en este trabajo se considera el constructivismo más convencional, según la definición de Sodupe (2002), que incluye a autores como Wendt (1999), Dessler (1989), Finnemore (1996), Jepperson (1996) y Katzenstein (1996).

En este sentido, Richard Price y Christian Reus-Smit (1998), definen tres presupuestos destacados sobre el constructivismo en relaciones internacionales que ayudan a esquematizar su lógica, en este marco más convencional de la teoría. El constructivismo para estos autores resalta por: I) El marco de estructuras de significados, que ejercen constreñimiento sobre los agentes. 2) Las identidades como constitutivas de los intereses de los agentes. 
3) La relación entre agentes y estructuras, centrada en prácticas que combinan condicionamientos con posibilidades de cambio estructural.

Este tipo de enfoque, como en la teoría de la estructuración de Giddens (2015), permite el diseño de orientaciones analíticas, así como definir conceptos operativos para pruebas empíricas en el marco de un proceso de investigación. En particular, en este trabajo y como modelo, consideramos los siguientes supuestos constructivistas desde una perspectiva china de las relaciones internacionales: I) Existen significados uniformes en la comunidad internacional, que tienen una función de control de ciertos Estados sobre otros. 2) La construcción de las identidades nacionales de los Estados responde a múltiple factores particulares. 3) Nuevas formas de cooperación pueden establecerse entre los Estados para el cambio estructural.

Desde esta perspectiva, el discurso chino en materia de política exterior viene a indicar el punto 3 , con nuevas formas de cooperación entre Estados y con dos tipos especiales de patrones de contenido que refieren al punto I y 2. Primero, se trata de promover un límite negativo de la cooperación (Lacan, 1998), sin contenido positivo para evitar replicar la función de control del supuesto número I. Esto significa centrarse más en lo que no se debe hacer que en lo que se debe hacer. Y, segundo, el discurso chino pondrá el foco estratégicamente en el respeto del supuesto número 2 como forma de cooperación. De esta manera, el discurso en política exterior de China manifestará repetidamente: "hemos asumido ante el mundo el solemne compromiso de nunca pretender la hegemonía" (Xi, 20I4: 315) y "ser respetuosos de la particularidad nacional en soberanía y seguridad e intereses del desarrollo" (Xi, 20I4: 62).

También, en este contexto, Yang y Zheng (20I7) se han referido al surgimiento de una nueva filosofía de gobernanza global y de características chinas, atendiendo a los principales desafíos de "fragmentación en la gobernanza y de competencia por la dominación sobre la producción de reglas de la gobernanza. A pesar de que existe una variedad de desafíos, la causa esencial de todos estos radica en la falta de consenso sobre una filosofía de la gobernanza" (Yang y Zheng, 20I7: 25). Por otra parte, desde un punto de vista de la teoría constructivista, el respeto por las particularidades de los Estados nacionales en la política exterior de China "permite abrir la caja negra de la formación de identidad e interés; los intereses de los Estados emergen de y son endógenos con respecto a la interacción con las estructuras" (Checkel, 1998: 326).

En este mismo sentido, pero ahora desde un punto de vista de orientación comunicativa (Hunston 1996; Lyons, 1995), el discurso de China ad- 


\section{Mariano Mosquera}

Principios y agenda en la política exterior china. Un análisis constructivista de los discursos de $\mathrm{Xi}$

quiere dos particularidades, relacionadas respectivamente con el punto I y 2 de nuestro modelo constructivista. A saber, un lenguaje prescriptivo de principios (Von Wright, 1963), pero abstractos para evitar condicionamientos (Laclau, 1987). Por otro lado, un lenguaje descriptivo de múltiples temas y actores (Ghanem, 2009), ya que el respeto por la particularidad nacional requiere de una multiplicidad de áreas de cooperación, en lugar de pocos espacios uniformes.

Este, al ser un enfoque que surge inductivamente del propio discurso a analizar, se vincula directamente con entender a la política exterior de China como diplomacia de características chinas. Sin embargo, el presente trabajo busca también controlar la aparición de referencias en el sentido de un discurso hegemónico chino, con intención de instalarse en el centro del sistema-mundo (Beeson, 2009; Liebman, 2005; Roy, 1997). Esto último se realiza a partir de la comparación con la política doméstica y partidaria y, específicamente, en la forma de control sobre las frecuencias y las relaciones de posibles normas e instituciones (Stoddart, 2007).

Así, este marco guiará el proceso de investigación en este trabajo, la búsqueda por la identificación de los principios y la agenda de la política exterior china; el diseño de la metodología, y, también, los criterios de interpretación de los resultados comparados. Es de destacar que suponer que China puede tener una orientación constructivista en su política exterior, contradice muchos antecedentes de este enfoque teórico, tal como manifiesta Vitelli (20I4).

\section{Metodología}

La metodología desarrollada incluye la construcción del corpus y el marco para la definición y análisis de los principios y agenda.

\subsection{Construcción del Corpus}

El corpus (Baker, 2006; Aarts y Meijs, 1984) analizado, se compone de la totalidad de discursos y artículos (79) del libro "La gobernación y administración de China" del Presidente de la
República Popular China, Xi (20I4). Se trata de discursos y artículos representativos tanto por autoridad como por actualidad, para "caracterizar un estado o variedad del lenguaje" (Sinclair, I99I: 17I). A continuación se detalla, en la Figura I, la composición de tokens de dicho corpus. 


\section{Figura 1}

Cantidad de tokens por tipo de política en el libro "La Gobernación y Administración de China” (Xi, 2014)

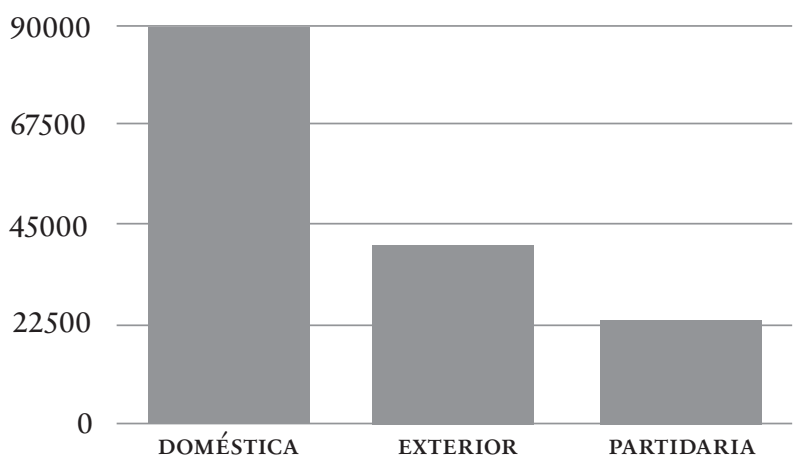

Fuente: elaboración propia en base a Xi (20I4)

Asimismo, dado el interés específi- por países o grupos de países, en el lico de esta investigación, se grafica la bro "La gobernación y administración composición de tokens en el marco de de China” de Xi (20r4). los discursos de política exterior china,

\section{Figura 2}

Cantidad de tokens por países o grupos de países en la política exterior en el libro "La Gobernación y Administración de China” (Xi, 2014)

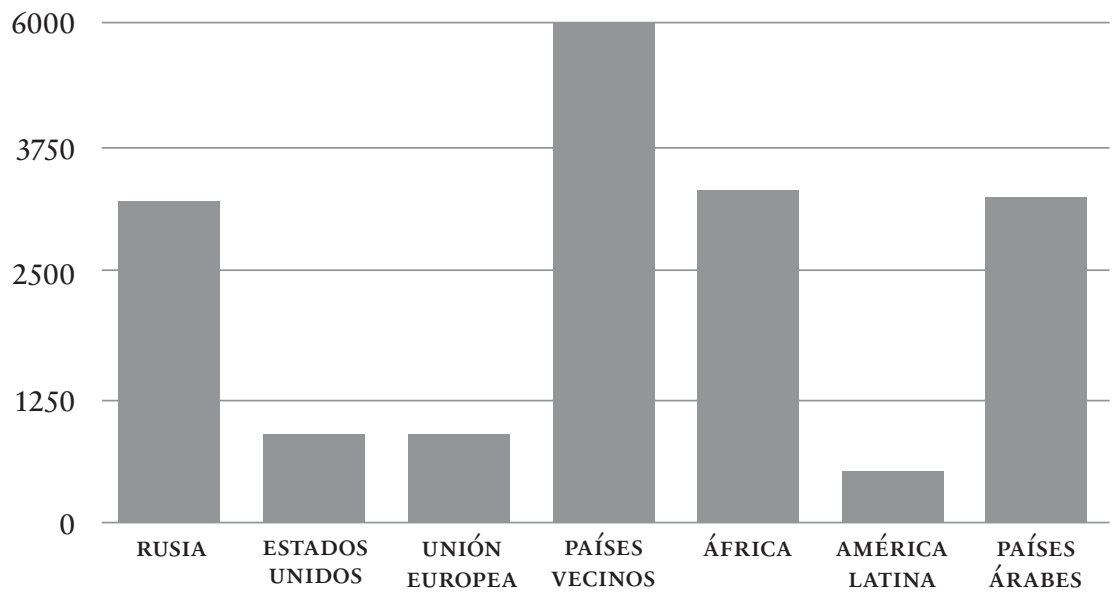

Fuente: elaboración propia en base a Xi (20I4) 


\section{Mariano Mosquera}

Principios y agenda en la política exterior china. Un análisis constructivista de los discursos de $\mathrm{Xi}$

La mayor cantidad de tokens se encuentra con los países vecinos de China. Luego, en un segundo nivel se encuentran Rusia, África y los países árabes. América Latina tiene el menor nivel de tokens, por debajo de Estados Unidos y de la Unión Europea. En este sentido, Su (20I7) ha dicho de la diplomacia de características chinas: "las mayores potencias son la clave, los vecinos la prioridad, los países en desarrollo su fundamento, y las plataformas multilaterales una importante instancia" (Su, 20I7: 26).

También, el libro de Xi (20I4) destaca por las frecuencias absolutas de algunos términos. Sin considerar palabras vacías (Biber, 1988), el término "desarrollo" es el más mencionado (I055 de FL), seguido por el término "Partido" (929 de FL), este último con más frecuencia que "China" (824 de FL).

\subsection{Definición y Análisis de Principios y Agenda}

Una multiplicidad de métodos se utilizan en este trabajo para la tarea de definición (identificación) y análisis de principios (Von Wright, 1963) y de agenda (Aruguete, 20I5). En particular $\mathrm{y}$ de forma progresiva, los siguientes seis puntos:

1. Se realiza un análisis de frecuencia léxica $(\mathrm{FL})$ para la priorización de términos normativos, temáticos y nombres propios de actores, considerando el $25 \%$ de la distribución superior de FL (Biber, 1988) en los distintos discursos y artículos en materia de política exterior, doméstica y partidaria. En este sentido, los análisis de FL han sido recurrentemente destacados para la identificación de jerarquías en el lenguaje (Puri, 2009).

2. Se trabaja con un control de ruido. Esto permite la identificación (y separación) de palabras vacías y de términos ambiguos de múltiples significados según el contexto (Stubbs, 1995).

3. Se realiza un control por categorías emergentes, con la identificación de principios, temas y actores de forma inductiva (Rodríguez Sabiote, Lorenzo Quiles y Herrera Torres, 2005) y por medio de la construcción de gazetteer o diccionarios para su clasificación. En el articulado de nuestra metodología este punto es importante, porque permite la identificación de términos en distinta morfología (verbos, sustantivos y adjetivos, de forma relacionada). Asimismo, esta tarea se realiza únicamente para la política exterior china, siendo las otras políticas (doméstica y partidaria) referencias para el estudio comparado.

4. Se trabaja con análisis de términos claves (keywords) para la identificación de los principios de política exterior de FL no esperadas, en comparación a la política do- 
méstica y a la política partidaria (Young, 1997).

5. Se realiza un análisis de concordancia Keyword In Context (Kw IC) para la construcción de principios y de agenda relacionales de alta FL, dentro del 25\% de la distribución superior de la política exterior, doméstica y partida$\mathrm{ria}^{5}$. La inclusión de un análisis de concordancia, en este trabajo, se debe a que consideramos que la concordancia "da acceso a muchos patrones importantes del lenguaje en el texto" (Sinclair, I99I: I70). Más aún, es en este tipo de corpus donde esperamos encontrar principios compuestos y en relación a múltiples temas y a distintos actores.

6. Se trabaja con análisis comparados (Peters, I998; Lijphart, I97I) para la interpretación de los resultados. Se utiliza el algoritmo de Kleinberg (1997) para el análisis de autoridad y el algoritmo de espacialización Force Atlas (Jacomy, Venturini, Heymann y Bastian, 20I4) para el análisis de distribución. De esta forma, primero, la autoridad en esquemas de visualización estará representada por el tamaño de los nodos.

\begin{abstract}
Este abordaje de análisis implica que el tamaño del nodo (o, en definitiva, de los términos) no solo se construye con su cantidad de conexiones, sino que también la autoridad de un término depende de la calidad de dichas conexiones (Kleinberg, 1997). Esto es por demás importante, porque nos permitirá estudiar términos de alta frecuencia y relacionamientos que se encuentran legitimados por otros términos de alta frecuencia y relacionamientos.
\end{abstract}

En el segundo caso, resaltamos que la vinculación por "cercanía” entre términos permite una identificación de determinadas discusiones en el discurso, como "unidad de segmentación" (Cortés Rodríguez, 20I4:59) y, por lo tanto, permite la identificación de los ejes articuladores de ciertos debates en el texto.

Finalmente, mencionar la construcción de un repositorio digital para asegurar la posibilidad de replicar el presente estudio.

5 Los términos de alta frecuencia (25\% superior) por política son sometidos a un análisis automático KwIC en el software SCP. Se relevan las frecuencias de términos en KWIC solo si se trata de términos de alta frecuencia. 


\section{Resultados}

A continuación presentamos los resultados del proceso de investigación, tanto por FL (y sus tareas vinculadas), como así también de forma relacional entre los términos de alta frecuencia.

\subsection{Definición de Principios por FL}

Según el análisis de FL y sus correlativas tareas de desambiguación, categorización emergente y análisis de términos claves, se pudieron identificar tres principios en la política exterior china:

1. Desarrollo pacífico

2. Beneficio mutuo

3. Proceso estratégico

Si bien el término "desarrollo" es de alta FL tanto en la política exterior como así también en la política doméstica y partidaria (I0,96; 5,77; I,24; respectivamente de $\mathrm{FL})^{6}$, el concepto de desarrollo pacífico es exclusivo de la política exterior. Se trata del principio central en materia de política exterior, ya que implica un progreso en condiciones de multilateralidad internacional, evitando la hegemonía de algún país sobre otros. Este es el sentido central en el discurso sobre la política exterior china. La FL de los términos "pacífico" y "paz" en la política exterior

6 Las frecuencias relativas están expresadas en base mil (I000). Se calculan sobre el total de términos: 89.734 para la política doméstica, 40.059 para la política exterior, y 23.390 para la política partidaria. es de I,I y 2,94, mientras que en la política doméstica es de 0,45 y 0,39. Estos términos no se encuentran dentro del $25 \%$ de la distribución superior de la política partidaria.

Por su parte, el principio de beneficio mutuo (FL de I,57 para "beneficio" y FL de 2,22 para "mutuo") implica una cooperación internacional que busca reducir las asimetrías entre los países de la relación. Varios términos son utilizados para contextualizar esta idea como un resultado "común" o "conjunto" (FL de I y 0,7 respectivamente, frente a un 0,59 solo para la aparición del término "común" en la política doméstica). Muchos de los términos asociados a este principio tienen aparición exclusiva en la política doméstica.

Finalmente, el último principio identificado se relaciona con términos más procesuales. Se trata de una estrategia (Oviedo, 2006) que busca articular los múltiples "intereses" (FL de I,74) de los países, con "respeto" (FL de $0,5)$ por lo "diverso" (FL de 0,65 ) de sus realidades nacionales. Una mayor dispersión de términos de alta frecuencia se relacionan con este principio, como "confianza" (FL de 0,9 ) y "diálogo" (FL de 0,6$)$ entre los más destacados.

Al tratarse de un principio procesual, la política doméstica y partidaria también refieren a términos como "intereses" (FL 0,89 y 0,77 , respectivamente), "diverso" (FL de I,55 y o,85) e incluso suman el término "peculia- 
ridades" (FL de 2,03 y 0,55, respectivamente para la política doméstica y partidaria). De esta forma, no se pudo identificar que el principio de proceso estratégico sea exclusivo de la política exterior por FL, aunque como se verá más adelante, este principio adquiere relaciones conceptuales diferentes entre las tres políticas.

Como control comparativo, destacamos que la política doméstica incluye con muy alta FL un principio de legalidad (FL de 2,II para "ley", FL de o,7 para "Constitución” e incluso una FL de 0,52 para el término "derechos”) que no tiene aparición de alta FL en la política exterior. Lo mismo sucede con principios morales que tienen aparición para la política doméstica (FL de I,24 para "valores" y FL de 0,45 para "moral") y que no se encuentran en la distribución superior de frecuencias de la política exterior.

Cierta similitud con la política doméstica tiene la política partidaria al incorporar legalidad $(0,85$ de FL) y moralidad (I,92 de FL). Aunque esta política suma "principios" de tipo "ideológicos", con "ideales" y "convicciones" (FL de 2,26 entre todos estos términos) que deben ser "estudiados" (FL de 2,9). Como exclusivo de la política partidaria, esta refiere a una lógica de "disciplina" (I,92 de FL) con "supervisión”, "control” (FL de I,2 y de 0,5I) y "responsabilidad" (FL de I,45). También es exclusivo de la política partidaria el principio de "servicio" (FL de 0,85 ) del Partido hacia la sociedad.

\subsection{Definición de Agenda por FL}

La agenda económica y en seguridad son ampliamente las de mayor frecuencia en la política exterior china, representando 4,52 y 4,6I de FL respectivamente. Muy alejada aparece la agenda social y la cultural (ambas con FL de $0,55)$. Destacamos aquí que la agenda económica posee, además, varios términos de alta frecuencia como son "comercio" o "inversión”, entre otros.

Respecto de los actores, en la política exterior de China existe un predominio de actores de Asia ("Asia", "Asia-Pacífico" y "ANSEA", con FL de 2,5; 0,87 y 0,5, respectivamente) y en menor medida actores de África (FL de $\mathbf{1}, 87)$ y de países en vías de desarrollo (FL de 0,55).

En términos comparados, la agenda económica y en seguridad también tienen alta FL en la política doméstica (FL de 2,4I y I,74), aunque no alcanzan el predominio relativo que tienen en la política exterior. Además, la agenda económica cuenta en la política doméstica, con otros términos de alta FL como "mercado", "producción” y "empresas". Sin embargo, la agenda en seguridad tiene relaciones conceptuales diferentes y las agendas social y cultural tienen una mayor preponderancia en la política doméstica (2,56 y I,33 de FL) que en la política exterior.

Además, en la política doméstica aparece una agenda sobre el régimen político socialista que es de muy alta

7 Asociación de Naciones del Sudeste de Asia. 
FL (el término "socialismo" con FL de 4,32 y el término "política” con FL de I,27), su "gobernación", "administración" e incluso su forma "democrática”. Estos temas no tienen aparición de alta FL en la política exterior. También, una agenda educativa, de la juventud y ambiental, son parte de la política doméstica de alta FL, no siendo parte de la política exterior.

En relación a los actores, el "Partido Comunista" tiene una FL muy alta de 5,66 en la política doméstica, seguido de "Taiwán” (con FL de 0,5I) como tema-actor de unificación nacional. Estos actores no tienen alta FL en la política exterior.

Finalmente, la política partidaria tiene una agenda muy alta de FL en la formación de sus "cuadros" políticos (6,62 de FL). Destaca también como agenda exclusiva partidaria la lucha contra la "corrupción” (FL de 3,37) y el centro del actor "Partido" (FL de I3,24) para referir a la agenda de su "administración", su "cultura" organizacional y la "educación” partidaria (FL de I,45; 0,38 y I,2, respectivamente). Todas estas son referencias sin aparición en la política exterior china.

\subsection{Análisis Relacional de \\ Principios y Agenda}

La política exterior china tiene un término articulador (de alta autoridad) que es el "desarrollo". La discusión de la política exterior de China gira en torno a este eje.

Este término se relaciona de forma cercana, por una parte, con el principio de desarrollo pacífico y con la agenda de seguridad. En particular, la seguridad en materia nuclear y en la región de Asia.

Por otra parte, también la agenda económica se encuentra cercana al término articulador de "desarrollo", aunque bien diferenciada del principio de desarrollo pacífico y de la agenda de seguridad. Particularmente, la agenda económica tiene vinculación con la temática social y está relacionada a la región Asia-Pacífico.

De forma más alejada a la discusión sobre el "desarrollo" se encuentra el principio de beneficio mutuo y el proceso estratégico, que aparecen muy vinculados entre ambos, ya que intercambian ciertos términos de influencia. Algunos términos como "confianza" destacan por su autoridad. Estos dos principios no aparecen tan vinculados a la discusión central sobre el "desarrollo" económico y pacífico, como se esperaba. En la Figura 3 puede visualizarse la relación de principios y agenda en la política exterior de China, con distribución basada en el algoritmo Force Atlas (Jacomy, Ven- 


\section{Figura 3}

Autoridad y distribución de términos de alta frecuencia en la política exterior en el libro La Gobernación y Administración de China (Xi, 2014)

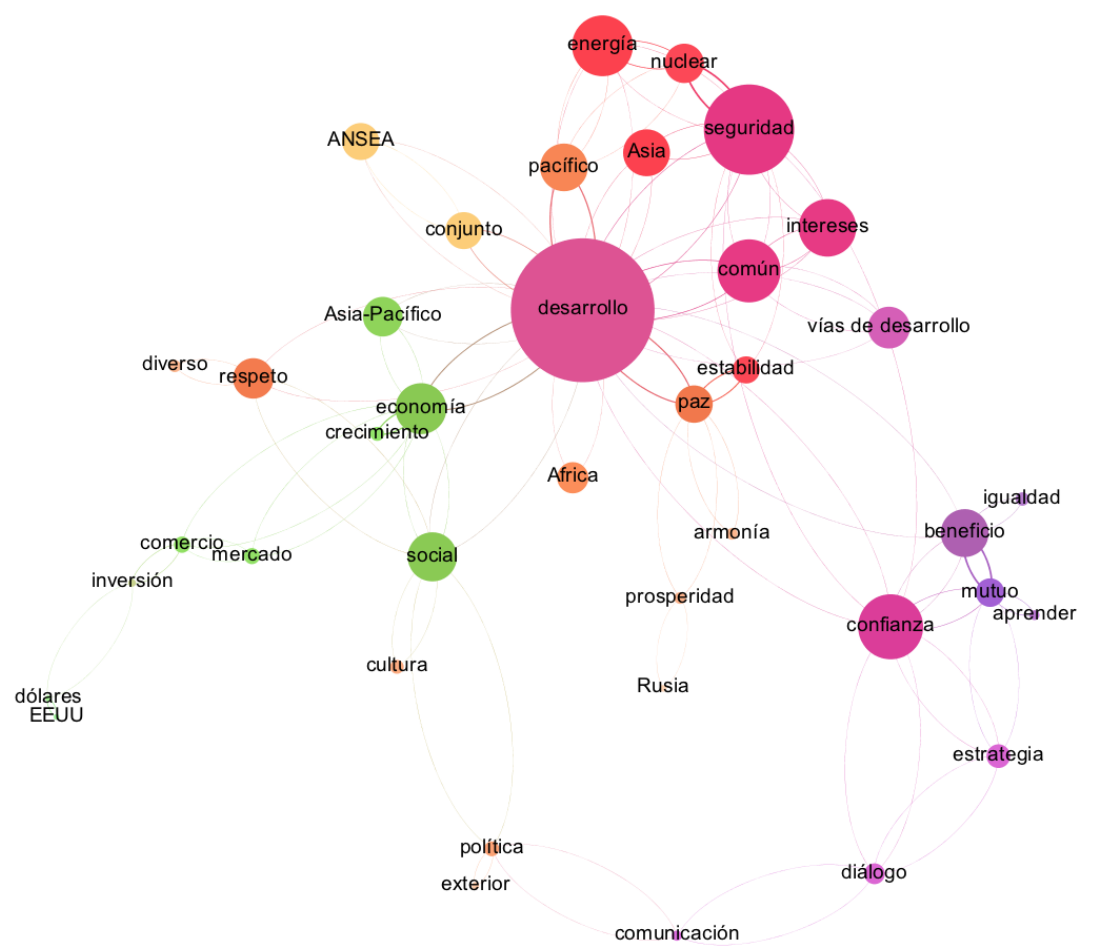

Fuente: elaboración propia en base a Xi (20I4)

turini, Heymann y Bastian, 20I4) y con tamaño de nodos por autoridad basada en el algoritmo de Kleinberg (1997).

Por su parte, y de forma comparativa, la distribución del discurso sobre la política doméstica adquiere distinta forma. El término "desarrollo" sigue siendo una autoridad que articula con la agenda de la "economía" y de la "seguridad”. Sin embargo, entre estos dos términos aparece el debate sobre la "propiedad". Además, pierde protagonismo la idea de desarrollo pacífico, siendo la seguridad cercana a términos como "leyes" y asociándose, por lo tanto, a la seguridad jurídica.

La economía se relaciona con el "socialismo" y sus "peculiaridades", siendo el término "socialismo" de muy alta autoridad. 


\section{Mariano Mosquera}

Principios y agenda en la política exterior china. Un análisis constructivista de los discursos de Xi

Otro término exclusivo de la po- dad", "estabilidad" y "producción") y lítica doméstica es la propia "políti- de la "cultura" (asociada a los "valores" ca”. Recordemos que la agenda política solo es referenciada como "política" "exterior" en el discurso sobre la misma política exterior, y con escasa autoridad para articular otros términos. También, con alta autoridad en la política doméstica, se destacan la agenda "social” (con "justicia", "equidel "socialismo"). El único actor dominante es el "Partido" en la política doméstica, con diversos satélites como "gobierno" y "administración”.

En definitiva, la política doméstica se centra en los significados políticos sobre el socialismo de características chinas, sobre su función económica,

\section{Figura 4}

Autoridad y distribución de términos de alta frecuencia en la política doméstica en el libro "La Gobernación y Administración de China”

(Xi, 2014)

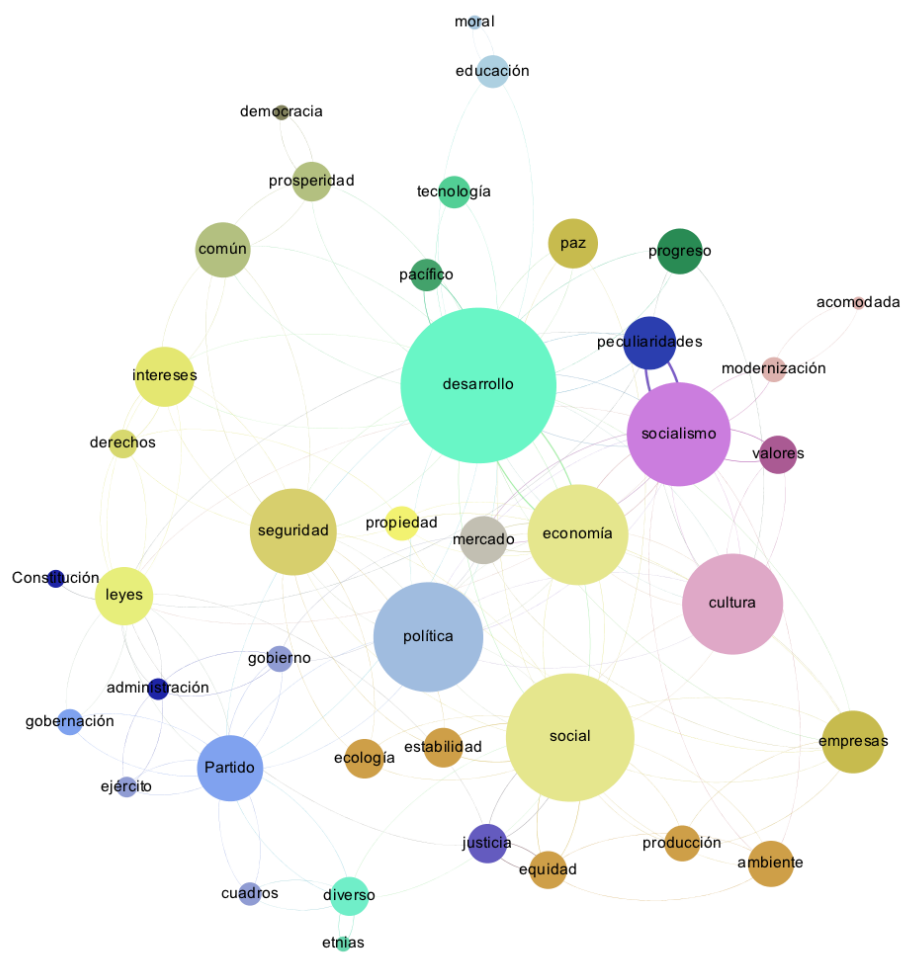

Fuente: elaboración propia en base a Xi (20I4) 
social y cultural, y sobre el liderazgo del partido y el rol de la ley en este proceso. En la Figura 4 puede observarse la autoridad y distribución de los términos de la política doméstica, como referencia comparativa con la política exterior china.

Finalmente, decimos que la política partidaria de China concentra en el actor "Partido" los principios y la agenda. En particular, los principios de "disciplina" y de "moralización" "admi- nistrativa" aparecen con alta autoridad y cercanos al debate sobre el "Partido". Por otra parte, la agenda de la "educación" de los "cuadros" partidarios se destaca especialmente, con alta autoridad y centralidad. En la Figura 5 puede verse la autoridad y distribución de términos de la política partidaria, para su comparación con el análisis relacional realizado sobre la política exterior de China.

\section{Figura 5}

Autoridad y distribución de términos de alta frecuencia en la política partidaria en el libro "La Gobernación y Administración de China" (Xi, 2014)

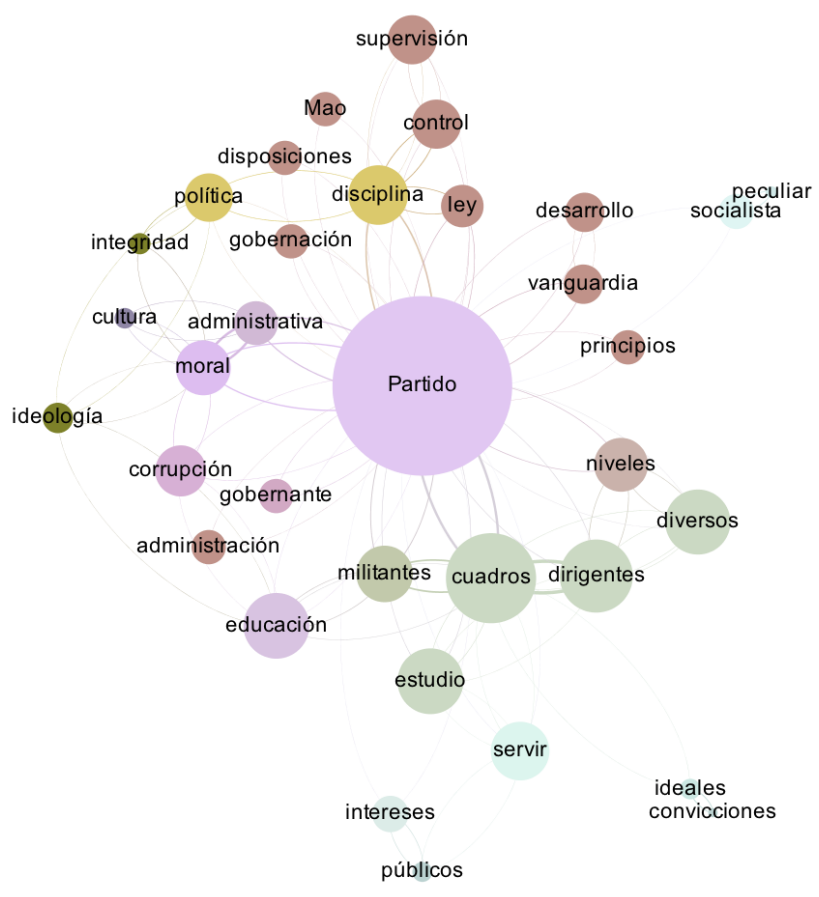

Fuente: elaboración propia en base a Xi (20I4) 


\section{Conclusiones y Discusión}

Presentamos como conclusión del presente trabajo de investigación las siguientes cinco afirmaciones sobre el discurso en política exterior del Presidente de China Xi (20I4):

I.- Solamente el principio de desarrollo pacífico se destaca con alta frecuencia, autoridad y relacionales conceptuales exclusivas en la política exterior de China.

2.- Los principios de beneficio mutuo y de proceso estratégico, y que también aportan al modelo constructivista, aparecen especialmente alejados del debate central sobre el desarrollo o compartiendo términos entre ellos o con la política doméstica y partidaria china. Esto parece debilitar, en el marco del presente análisis, la fuerza de estos dos principios.

3.- En el sentido de la primera y segunda afirmación, la política exterior de China se destaca más por fomentar la paz como agenda de seguridad que por encontrar condiciones para reducir asimetrías en la cooperación entre países. Esto último incluso en un contexto de concentración económica de la agenda de política exterior, donde principios como el de beneficio mutuo deberían ser más relevantes.

4.- La agenda de actores en la política exterior china también es concentrada en países vecinos de China. Esto sucede tanto en temas económicos como de seguridad.

5.- A nivel comparativo, la política doméstica y partidaria china poseen principios que refieren a normas o instituciones de control, no estando esto presente en la política exterior. Asimismo, la política doméstica es más amplia (múltiple) que la política exterior china.

De esta forma, la hipótesis de esta investigación se confirma solo parcialmente, ya que la orientación contra-hegemónica y de respeto por la particularidad de los países tiene aparición, aunque con restricciones y en el marco de una agenda temática y de actores concentrada. Así, una orientación de tipo constructivista en la política exterior china solamente puede afirmarse parcialmente, en el marco de los discursos de Xi (20I4).

Como discusión final de este trabajo presentamos, a continuación, dos ejes que consideramos como un aporte a los debates presentes y futuros sobre el discurso de la política exterior de China.

Primero, si bien el discurso de la diplomacia de características chinas fomenta, en su contenido, un mundo multipolar y se opone a una orientación de tipo hegemónica, la construcción discursiva china, en su forma, posee falencias que pueden afectar el propósito comunicativo o de entendimiento del propio discurso. Se trata, más bien, de restricciones por la concentración de la agenda y el predominio de algunos principios por sobre otros. Es decir, si bien el discurso en política exterior no incorpora direc- 
tamente construcciones normativas $o$ institucionales de control que puedan indicar orientación hegemónica, las propias restricciones mencionadas podrían inducirla, aunque esta última orientación no fuera el propósito comunicativo del productor del discurso. También, otra perspectiva que busque indagar sobre los "motivos" del discurso chino podría suponer un propósito hegemónico construido de manera indirecta en el discurso; es decir, no el contenido, sino a partir de su forma.

Segundo, y finalmente, las futuras agendas de investigación en relaciones internacionales deben considerar una posible orientación en política exterior de la teoría constructivista, en particular con el caso de la diplomacia china.

\section{BibliografíA}

Aarts, J. y Meijs, W. (1984). Corpus Linguistics. Amsterdam: Rodopi.

Arrighi, G. y Drangel, J. (1986). “The stratification of the world-economy: an exploration of the semiperipheral zone". Review (Sage Publications), vol. X, no. I (Verano 1986), pp. 9-74.

Aruguete, N. (2015). El Poder de la Agenda. Política, Medios y Público. Buenos Aires: Biblos.

Bach, K. (200I). "Semantically Speaking”, en Kiefer, F.; Kenesei, I. y Harnish, R. (eds.), Perspectives on Semantics, Pragmatics, and Discourse. Amsterdam: John Benjamins, pp. I47-I70.

Baker, P. (2006). Using corpora in discourse analysis. London: Continuum.

Beeson, M. (2009). "Hegemonic Transition in East Asia? The Dynamics of Chinese and American Power". Review of International Studies, vol. 35, no. I, pp. 95-II2. Recuperado el 20 de Julio de 2017 de http://dx.doi.org/IO.IOI7/ So26021050900834I
Beutel, F.K. (1965). Democracy or the scientific method in law and policy making. San Juan de Puerto Rico: UPR.

Biber, D.; Conrad, S. y Reppen, R. (1998). Corpus linguistics. Investigating language structure and use. Cambridge: Cambridge University Press.

Biber, D. (1988). Variation across speech and writing. Cambridge: cup.

Checkel, J. (1998). "The constructivist turn in international relations theory". World Politics, vol. 50, no. 2, pp. 324-348. Corkin, L. (20I4). "China's Rising Soft Power: The Role of Rhetoric in constructing China-Africa relations”. Revista Brasileira de Política Internacional, no. 57, pp. 49-72.

Cortés Rodríguez, L. (20I4). "Sobre inicios y cierres en el discurso político”. Estudios Filológicos, no. 53, pp. 55-69.

Dessler, D. (1989). "What's at Stake in the Agent-Structure Debate?” International Organization, vol. 43, no. 3, pp 44I-473. Dworkin, R. (20I4). Los derechos en serio. Barcelona: Ariel Derecho. 
Mariano Mosquera

Principios y agenda en la política exterior china. Un análisis constructivista de los discursos de $\mathrm{Xi}$

Finnemore, M. (1996). National Interests in International Society. New York: Cornell University Press.

Ghanem, S. (2009). "Filling in the Tapestry: The Second Level of Agenda Setting", en McCombs, M.; Shaw, D.L. y Weaver, D. (eds.), Communication and Democracy. Exploring the Intellectual Frontiers in Agenda Setting Theory. Mahwah: Lawrence Erlbaum, pp. 3-I4.

Giddens, A. (20I5). La constitución de la sociedad. Bases para la teoría de la estructuración. Buenos Aires: Amorrortu.

Henderson, W. y Hewings, A. (1990). "Language and Model Building?”, en Dudley-Evans, A. y Henderson, W. (eds.), The Language of Economics: The Analysis of Economics Discourse. London: Macmillan y British Council, pp. 43-54.

Hopf, T. (1998). "The Promise of Constructivism in International Relations Theory". International Security, vol. 23, no. I, pp. I7I-200.

Hunston, S. (1996). "Evaluation and Organization in a Sample of Written Academic Discourse", en Coulthard, M. (ed.), Advances in Written Text Analysis. London: Routledge, pp. 19I-2I8.

Ikenberry, J. (2008). "The Rise of China and the Future of the West". Foreign Affairs (Council on Foreign Relations), vol. 87, no I, pp. 23-37.

Jacomy, M.; Venturini, T.; Heymann, S. y Bastian, M. (20I4). "ForceAtlas2, a Continuous Graph Layout Algorithm for Handy Network Visualization Designed for the Gephi Software". PLoS ONE, vol. 9, no. 6: e98679. Recuperado I6 de junio de 2017 de doi:I0.I37I/ journal.pone.0098679
Jenkins, R. (20I2). "Latin America and China - a new dependency?" Third World Quarterly (Routledge), vol. 33, no. 7, pp. I337-I358.

Jepperson, R.L. (1996). "Norms, Identities, and Security in the Contemporary World System", en Katzenstein, P.J. (ed.), The Culture of National Security. Norms and Identity in World Politics. New York: Columbia University Press, Ch. 2.

Katzenstein, P.J. (1996). The Culture of $\mathrm{Na}$ tional Security. Norms and Identity in World Politics. Nueva York: Columbia University Press.

Keohane, R. y Nye J. (1989). Power and Interdependence: World Politics in Transition. Boston: Brown, Little and Company.

Keohane, R. (2006). Liberalism, Power and Governance in a Partially Globalized World. Beijing: Peking University Press.

Kleinberg, J.M. (1997). "Authoritative Sources in a Hyperlinked Environment". Proceedings of the ACM-SIAM Symposium on Discrete Algorithms.

Kotschwar, B. (20I4). "China's economic influence in Latin America". Asian Economic Policy Review (Japan Center for Economic Research), vol. 9, no. 2, pp. 202-222.

Lacan, J. (1998). The Seminar of Jacques Lacan: The Four Fundamental Concepts of Psychoanalysis. New York: W. W. Norton Company.

Laclau, E. y Mouffe, Ch. (1987). Hegemonía y Estrategia Socialista. Hacia una Radicalización de la Democracia. Madrid: Siglo XXI Editores.

Liebman, A. (2005). "Trickle-down Hegemony? China's Peaceful Rise and 
Dam Building on the Mekong”. Contemporary Southeast Asia, vol. 27, no. 2, pp. 28I-304. Recuperado 3 de Agosto de 2017 de http://www.jstor.org/ stable/25798737

Lijphart, A. (I97I). "Comparative Politics and the Comparative Method". American Political Science Review (American Political Science Association), vol. 65, no. 3, pp. 682-693.

Liu, J. (20I7). "Major-country diplomacy with Chinese characteristics reflects trends of the times". China International Studies, May/June 20I7, pp. 28-44.

Lyons, J. (1995). Linguistic Semantics. An Introduction. Cambridge: Cambridge University Press.

Morales Ruvalcaba, D. (2017). "El fin del ciclo hegemónico de Estados Unidos". Foreign Affairs Latinoamérica. Recuperado 23 de Junio de 2017 de http:// revistafal.com/el-fin-del-ciclo-hegemonico-de-estados-unidos/

Morales Ruvalcaba, D. (20I5). Poder, estructura y hegemonía: pautas para el estudio de la gobernanza internacional. Vol. I: Índice de Poder Mundial. Guadalajara: UG.

Nye J. (2004). Soft Power: The Means to Success in World Politics. New York: Public Affairs.

Oviedo, D. (2006). "China: Visión y Práctica de sus llamadas Relaciones Estratégicas". Estudios de Asia y África, vol. 4I, no. 3, pp. 385-404.

Pastrana, E. y Vera, D. (20I7). “Transición de poder y orden mundial: el ascenso global de China y su proyección”, en Pastrana, E. y Gehring, H. (eds.), $L a$ proyección de China en América Latina y el Caribe. Bogotá: Pontificia Universidad Javeriana, pp. 23-72.

Peters, B. Guy (1998). Comparative Politics: Theory and Methods. New York: New York University Press.

Price, R. y Reus-Smit, Ch. (1998). "Dangerous Liaisons? Critical International Theory and Constructivism". European Journal of International Relations, vol. 4, no. 3, pp. 259-294.

Price, R. y Tannenwald, N. (1996). “Norms and Deterrence: The Nuclear and Chemical Weapons Taboos”, en Katzenstein, P.J. (ed.), The Culture of $\mathrm{Na}$ tional Security. Norms and Identity in World Politics. Nueva York: Columbia University Press, pp. II4-I52.

Puri, Ishan S. (2009). "Lexical Distributions and Electronic Literacy: A Corpus Linguistic Analysis of Textual Richness". Working paper. Recuperado 8 de Septiembre de 2017 de https:// ssrn.com/abstract=I426622 o de http:// dx.doi.org/IO.2I39/ssrn.I426622

Rodríguez Sabiote, C.; Lorenzo Quiles, O. y Herrera Torres, L. (2005). “Teoría y Práctica del Análisis de Datos Cualitativos. Proceso General y Criterios de Calidad". Revista Internacional de Ciencias Sociales y Humanidades, vol. 15, no. 2, pp. 133-154. Roy, D. (1996). "The China Threat". Asian Survey, vol. 36, no. 8, pp. 75877. Recuperado 9 de Julio de 2017 de doi: $10.2307 / 2645437$

Ruggie, J.G. (1998). "What makes the world hang together? Neo-utilitarianism and the social constructivism challenge". International Organization, vol. 52, no. 4, pp. $855-885$. 


\section{Mariano Mosquera}

Principios y agenda en la política exterior china. Un análisis constructivista de los discursos de $\mathrm{Xi}$

Serbín, A. (20I7). "China y América Latina y el Caribe frente a un cambio de ciclo: narrativas y estrategias", en Pastrana, E. y Gehring, H. (eds.), La proyección de China en América Latina y el Cari$b e$. Bogotá: Pontificia Universidad Javeriana/Fundación Konrad Adenauer, pp. 73-97.

Sinclair, J. (199I). Corpus Concordance Collocation. Oxford: Oxford University Press.

Sodupe, K. (2002). "Del tercer al cuarto debate en las relaciones internacionales". Revista Española de Derecho Internacional, vol. 54, no. I, pp. 65-93.

Stoddart, M. (2007). "Ideology, Hegemony, Discourse: A Critical Review of Theories of Knowledge and Power". Social Thought \& Research, no. 28, pp. 19I-225.

Stubbs, M. (1995). "Collocations and semantic profiles: On the cause of the trouble with quantitative studies". Functions of Language, no. 2, pp. 23-55.

$\mathrm{Su}, \mathrm{G}$. (2017). "The great historic journey of Chinese diplomacy". China International Studies, November/December 20I7, pp. 5-39.

Vittelli, M. (20I4). "Veinte Años de Constructivismo en Relaciones Internacionales. Del Debate Metateórico al Desarrollo de Investigaciones Empíricas. Una Perspectiva sin un Marco de Po- lítica Exterior”. PostData, vol. 19, no. I, pp. I29-I62.

Von Wright, G. (1963). Norm and action. A logical enquiry. Londres: Routledge and Kegan Paul.

Wallerstein, I. (2003a). El moderno sistema mundial. Tomo I: la agricultura capitalista y los orígenes de la economía-mundo europea en el siglo xvI. México D.F.: Siglo XXI.

Wallerstein, I. (2003b). El moderno sistema mundial. Tomo II: El mercantilismo y la consolidación de la economía-mundo europea 1600-1750. México D.F.: Siglo XXI.

Wendt, A. (1999). Social Theory of International Politics. New York: Cambridge University Press.

$\mathrm{Xi}$, Jinping (20I4). La gobernación y administración de China. Beijing: Ediciones en Lenguas Extranjeras Cía. Ltda.

Yang, J. y Zheng, Y. (2017). "Global governance of new frontiers: China's perspective". China International Studies, September/October 20I7, pp. 24-44.

Young, S. (1997). Corpus-Based Methods in Language and Speech Processing (Mathematics and Its Applications). Dordrecht: Kluwer.

Yu, J. (20I7). "China's Rise: Historical inevitability and realistic path". China International Studies, May/June 20I7, pp. I34-I40. 


\section{Anexo}

Tabla

Capítulos y discursos de política exterior en el libro "La Gobernación y Administración de China” (Xi, 2014)

\begin{tabular}{|c|c|}
\hline CAPÍTULO DE POLÍTICA EXTERIOR & TÍTULO DEL DISCURSO \\
\hline \multirow[t]{5}{*}{$\begin{array}{l}\text { XI. SEGUIR EL CAMINO DEL } \\
\text { DESARROLLO PACÍFICO. }\end{array}$} & $\begin{array}{l}\text { Coordinar la situación nacional e } \\
\text { internacional y fortalecer la base } \\
\text { del camino del desarrollo pacífico. }\end{array}$ \\
\hline & $\begin{array}{l}\text { Abrir un nuevo camino en la coo- } \\
\text { peración de beneficio mutuo. }\end{array}$ \\
\hline & $\begin{array}{l}\text { Persistir en la concepción de la } \\
\text { seguridad nuclear racional, coordi- } \\
\text { nada y equilibrada. }\end{array}$ \\
\hline & $\begin{array}{l}\text { Los intercambios y el aprendizaje } \\
\text { mutuos dan colorido y enriquecen } \\
\text { las civilizaciones. }\end{array}$ \\
\hline & $\begin{array}{l}\text { El compromiso de China con el } \\
\text { desarrollo pacífico. }\end{array}$ \\
\hline \multirow[t]{3}{*}{$\begin{array}{l}\text { XII. IMPULSAR LA ESTRUCTURACIÓN } \\
\text { DE UN NUEVO TIPO DE RELACIONES } \\
\text { ENTRE PAÍSES GRANDES. }\end{array}$} & $\begin{array}{l}\text { Corresponder a la tendencia de la } \\
\text { época, promover el desarrollo pací- } \\
\text { fico del mundo. }\end{array}$ \\
\hline & $\begin{array}{l}\text { Estructurar un nuevo modelo de } \\
\text { relaciones de países grandes entre } \\
\text { China y Estados Unidos. }\end{array}$ \\
\hline & $\begin{array}{l}\text { Tender un puente de amistad y } \\
\text { cooperación sobre el continente } \\
\text { eurasiático. }\end{array}$ \\
\hline
\end{tabular}




\section{Mariano Mosquera}

Principios y agenda en la política exterior china. Un análisis constructivista de los discursos de Xi

\begin{tabular}{|c|c|}
\hline CAPÍTULO DE POLÍTICA EXTERIOR & TÍTULO DEL DISCURSO \\
\hline \multirow{3}{*}{$\begin{array}{l}\text { XIII. CUMPLIR COMO ES DEBIDO LA } \\
\text { LABOR DIPLOMÁTICA CON LOS PAÍSES } \\
\text { VECINOS. }\end{array}$} & $\begin{array}{l}\text { Construyamos la "Franja Económi- } \\
\text { ca de la Ruta de la Seda". }\end{array}$ \\
\hline & $\begin{array}{l}\text { Construyamos conjuntamente la } \\
\text { "Ruta Marítima de la Seda" del siglo } \\
\text { xxI. }\end{array}$ \\
\hline & $\begin{array}{l}\text { Diplomacia con los países vecinos } \\
\text { sobre la base de la amistad, sinceri- } \\
\text { dad, reciprocidad e inclusividad. }\end{array}$ \\
\hline \multirow{3}{*}{$\begin{array}{l}\text { XIV. FORTALECER Y DESARROLLAR } \\
\text { LA UNIDAD Y COOPERACIÓN CON LOS } \\
\text { PAÍSES EN VÍAS DE DESARROLLO. }\end{array}$} & $\begin{array}{l}\text { Ser amigos confiables y socios sin- } \\
\text { ceros para siempre. }\end{array}$ \\
\hline & $\begin{array}{l}\text { Impulsar la realización de un nuevo } \\
\text { y mayor desarrollo de las relaciones } \\
\text { entre China y América Latina. }\end{array}$ \\
\hline & $\begin{array}{l}\text { Desplegar el espíritu de la Ruta de } \\
\text { la Seda y profundizar la coopera- } \\
\text { ción China-Árabe. }\end{array}$ \\
\hline \multirow[t]{6}{*}{$\begin{array}{l}\text { XV. PARTICIPACIÓN ACTIVA EN LOS } \\
\text { ASUNTOS MULTILATERALES. }\end{array}$} & $\begin{array}{l}\text { Aunemos esfuerzos en la coope- } \\
\text { ración por alcanzar el desarrollo } \\
\text { común. }\end{array}$ \\
\hline & $\begin{array}{l}\text { Creemos juntos un futuro mejor } \\
\text { para Asia y el mundo. }\end{array}$ \\
\hline & $\begin{array}{l}\text { Mantengamos y desarrollemos jun- } \\
\text { tos una economía mundial abierta. }\end{array}$ \\
\hline & $\begin{array}{l}\text { Fomentemos el "espíritu de Shan- } \\
\text { ghái” y el desarrollo conjunto. }\end{array}$ \\
\hline & $\begin{array}{l}\text { Profundizar la reforma y apertura } \\
\text { y crear una región Asia-Pacífico } \\
\text { hermosa. }\end{array}$ \\
\hline & $\begin{array}{l}\text { Nuevo enfoque para la cooperación } \\
\text { de seguridad en Asia. }\end{array}$ \\
\hline
\end{tabular}

Fuente: "La gobernación y administración de China" (Xi, 20I4) 